\title{
TUEURS À GAGES \\ ET CONFLITS INTERPERSONNELS \\ DANS LE BRÉSIL URBAIN : \\ D'ANCIENNES PRATIQUES \\ RECONSIDÉRÉES
}

par César BARREIRA

RÉSUMÉ

Dans les sociétés contemporaines, les conflits interpersonnels se présentent sous différentes configurations. Leurs cadres d'affrontement et de résolution se diversifient tandis que la participation de tueurs à gages dans ces pratiques conflictuelles gagne du terrain. Les homicides en relation à la pratique du meurtre sur commande apparaissent dans les bagarres politiques, les amours non partagées, les querelles de familles, les personnes "suspectes", etc. Le meurtre sur commande se définit par la présence de deux personnages : le tueur à gages qui exécute l'action, et le commanditaire qui subventionne le crime en payant pour la pratique délictueuse. L'article analyse ces pratiques et cherche à en comprendre les changements et les continuités dans un contexte urbain contemporain. Les articles divulgués par la presse et les entretiens réalisés avec des personnages liés directement ou indirectement à ce système montrent les transformations des pratiques du meurtre sur commande et leurs rapports avec les sociabilités quotidiennes du monde urbain. Il est mis en évidence le fait que non seulement les donneurs d'ordres et les meurtriers sur commande mais aussi les victimes subissent des mutations. Celles-ci vont être constituées dans un contexte contemporain de violence diffuse et, fondamentalement, comme conséquence de l'accroissement de l'utilisation des tueurs à gages. Brésil.

Mots clés: Tueur à gages, Conflits interpersonnels, Violence urbaine,

\section{SUMMARY}

In present-day societies inter-personal conflicts assume many different configurations. The contexts in which confrontation and resolution take place are multiple, while, at the same time, the number of professional killers is increasing. Homicides related to the practice of resorting to gunmen are found in political campaigns, unrequited love situations, family feuds, "suspected" persons, etc. A planned murder involves two persons : the gunman who commits the crime and the interested party who pays the gunman for the

1. Traduit par Agnès Clerc-Renaud.

Cahiers internationaux de Sociologie, Vol. CXXVII [315-337], 2009 
criminal act. This article analyses these practices and endeavors to understand the changes and continuity in contemporary urban society. The articles divulged by the press and interviews with people directly or indirectly involved in the cases reveal changes in gunmen's practices and their links with the social interaction in the urban world. We demonstrate that not only the hired killers and their sponsors are changing but so are the victims. These changes are shaping this practice into a kind of diffuse violence which basically results in a growing number of people resorting to the use of professional killers.

Key words: Gunman, Interpersonal Conflicts, Urban Violence, Brazil.

Chaque jour ou presque, les principaux journaux nationaux du Brésil publient des articles sur les crimes de tueurs à gages. Ils remettent en scène un personnage historique de la société brésilienne, originaire de l'espace social agraire: le tueur à gages ou meurtrier à louer.

Le meurtre sur commande se distingue sociologiquement d'autres crimes par la présence de deux personnages : l'exécuteur d'une action dénommée "besogne " ou "travail " et le donneur d'ordre, qui subventionne le crime en payant pour la pratique délictueuse. Dans cette perspective, le tueur à gages - l'auteur matériel - qui exécute l'action et le donneur d'ordre - l'auteur intellectuel - qui la commande constituent les chevilles ouvrières qui définissent le « crime sur ordonnance ".

"Dix-neuf assassinats. Dix-neuf victimes de coups de feu tirés par des meurtriers à louer. Tel est le bilan des meurtres sur commandes survenus cette année dans le seul État du Ceará, contre 13 enregistrés en 2006 pour la même période, soit une augmentation de $46,15 \%$ " (journal Diario do Nordeste, 11 juin 2007).

"Cinq personnes ont été emprisonnées à Morada Nova, à $170 \mathrm{~km}$ de Fortaleza, accusées d'implication dans un meurtre sur commande survenu le mois dernier. En plus des auteurs matériels du crime, José Narcélio de Oliveira, 20 ans, et un adolescent de 17 ans (témoins passés aux aveux), ont été emprisonnés le donneur d'ordre de la mort du boucher et travailleur rural Raimundo Vandeilson Rabelo, connu sous le nom de "Veio Duildo" ainsi que l'intermédiaire du crime, Adilson Ferreira, 33 ans. Ce dernier a eu selon la police un rôle important dans l'affaire en réalisant la passation du contrat des tueurs à gages pour la somme de 1000 reais. D'après la police, Adailton a reçu la somme de 500 reais pour le travail " (journal O Povo, 4 février 2007).

La présente étude de la relation entre les pratiques de meurtre sur commande et la violence quotidienne diffuse et récurrente basée sur des mésententes personnelles a surgi quasi spontanément de recherches réalisées dans les médias. Les articles diffusés par la presse et les entretiens réalisés avec des personnages liés directement ou indirectement au système du meurtre sur commande montrent 
des transformations de ces pratiques, en lien profond avec les sociabilités quotidiennes du monde urbain.

L'analyse des différentes formes d'expression de la violence dans les sociétés contemporaines met en relief une nouvelle configuration des conflits sociaux ainsi qu'une diversification du cadre d'affrontement et de gestion des conflits interpersonnels. Harmonie et dysharmonie, amour et haine sont des ingrédients du processus de sociabilité qui montrent les conflits interpersonnels comme marquant le tissu des relations sociales actuelles.

Au sein de cette configuration, une donnée incline en ce sens : la récurrence de pratiques sociales violentes dans la façon d'affronter ou de gérer les conflits, ou, comme le formule le sens commun, de tenter de les résoudre. De façon récurrente, les conflits interpersonnels continuent à être gérés par l'usage de la violence physique. En ce sens, la participation de tueurs à gages dans les pratiques conflictuelles gagne du terrain, et montre un cadre de résolution des disputes qui ne fait pas usage du dialogue, ni de la parole, ni des possibilités d'accord ou de conciliation. Le choix consiste dans l'élimination de l' "ennemi » ou de l' "opposant».

Les homicides en relation à la pratique du meurtre sur commande apparaissent dans diverses situations : les bagarres politiques, les amours non partagées, les querelles de familles, contre les épouses infidèles, les "voisins indésirables » les personnes "suspectes ", etc.

Ces scènes sont justifiées par un discours élaboré en faveur de l'usage de la violence comme d'une asepsie du social : « il y a des gens qui doivent vraiment mourir ", " elle n'a jamais rien valu, elle n'était ni une bonne épouse, ni une bonne mère ", "certaines personnes n'arrivent plus à vivre en société, pourquoi vivraient-elles? »

L'usage récurrent de la violence est alors légitime : la violence est combattue par la violence et les disputes personnelles ou les rixes peuvent être résolues par des agressions morales ou physiques, ce qui fournit aux relations sociales un substrat dont l'intolérance et la banalisation de la vie sont les principaux ingrédients.

Dans certains cas, la sanction publique est limitée, et il existe manifestement une relative acceptation de la violence, pourtant associée à un autre univers de significations sociales. Pour une partie de la population, les actions des tueurs à gages, justiciers ou vengeurs ne sont pas condamnées totalement. Dans certains cas, elles sont même justifiables, ce qui désavoue l'attitude bipolaire vis-à-vis du bon et du mauvais, du vrai et du faux, du bien et du mal, du légal et de l'illégal.

En l'absence de contrôle et de discipline, la peur, la précaution et l'autodéfense gagnent de l'importance devant un monopole désordonné de la violence étatique. 
Des espaces sociaux "non pacifiés ", voire étrangers au processus civilisateur montré par Norbert Elias se font jour, qui manifestent un cadre de violence diffuse et une banalisation de la vie.

Cet article prétend analyser les diverses nuances que revêtent les pratiques des meurtriers à louer. Il vise à en comprendre les changements et les continuités dans le nouveau contexte social et économique qui caractérise le système du meurtre sur commande.

Cette étude est basée sur des données empiriques provenant d'entretiens réalisés avec des personnes accusées d'être des tueurs à gages ou meurtriers à louer. Les entretiens ont été réalisés avec des personnes emprisonnées, des personnes sorties de prison ayant déjà accompli leur peine ou encore pour n'avoir jamais été arrêtées. De ce fait, les entretiens ont eu lieu selon les cas à l'intérieur des prisons, aux domiciles des personnes ou dans des lieux publics. Tous les entretiens ont été obtenus par l'intermédiaire de tiers : avocats, prêtres ou amis qui entretenaient des relations d'amitié ou d'influence avec les informateurs. On s'appuiera aussi sur des articles relatifs aux conflits interpersonnels et aux crimes sur ordonnance publiés entre 2001 et 2007 par les principaux journaux quotidiens de l'État du Ceará ${ }^{1}$ : O Diario do Nordeste et O Povo.

\section{VISIBILITÉ ET MÉLANGE DES CODES ET DES VALEURS}

Actuellement, les «contrats " de tueurs à gages gagnent de plus en plus de visibilité pour résoudre un vaste éventail de conflits sociaux. Ils impliquent des voisins, des conjoints, des parents, des amis, c'est-à-dire des situations sociales qui incorporent des relations de proximité physique et sociale. À partir de l'action des tueurs à gages dans des conflits sociaux, impliquant des pratiques de "justices », ce cadre sera analysé en prenant pour élément central spécifique sa base de tolérance et d'acceptation sociale. Dans le mélange des codes et des valeurs se dessinent les contours d'une façon particulière de faire justice, celle d'une "justice parallèle ».

Les justifications que la presse et le sens commun donnent de ces crimes sont diverses et comprennent des motifs perçus comme "futiles et banals». Chaque homicide fait surgir des spéculations comportant un ample éventail de possibilités quant aux motifs de l'acte criminel. Sont cependant privilégiées les versions de «brûlage

1. L'État du Ceará est l'un des États fédérés de la République des États-Unis du Brésil. 
d'archives ${ }^{1}$, de querelles de familles, de dettes non acquittées, de vengeances, de bagarres politiques et de problèmes amoureux.

De façon fréquente et récurrente, certains homicides sont définis par les médias comme des "crimes ayant les caractéristiques du meurtre sur commande". Ils comportent en principe des aspects spécifiques. Ces homicides sont commis en utilisant la tactique de l'embuscade, la victime étant attaquée par surprise. Plusieurs coups de feu sont tirés, en général dans la tête sans qu'aucune dispute ne précède l'assassinat. Ces actes se déroulent généralement dans des lieux publics. L'auteur matériel du crime ne fait pas partie du réseau social de la victime. Enfin, le véhicule utilisé pour pratiquer l'action meurtrière est une motocyclette et l'action est accomplie par deux personnes: le passager et le conducteur, ce dernier apparaissant comme complice.

La diversité des motifs complique la compréhension de la nature du crime commandité de la part du pouvoir judiciaire, des organes de sécurité et de la presse. Cependant le phénomène s'inscrit dans un réseau de valeurs et de représentations sociales riche et complexe. Il est important de souligner que les meurtres sur ordonnance comportent un mélange de réalité et de fiction ${ }^{2}$ qui stimulent l'imagination sociologique, pour l'analyse du personnage principal : le tueur à gages.

Ce dernier peut être vu comme un agent social "décalé ", "hors du temps » et "hors de l'espace » dans la mesure où il reproduit la situation d'une autre époque, durant laquelle ces pratiques étaient courantes. Actuellement, la pratique du meurtre sur commande se professionnalise et les tueurs à gages ne maintiennent plus de relations de dépendance et de sujétions vis-à-vis des grands propriétaires terriens. Les liens affectifs à leur réseau d'appartenance disparaissent également, ce qui met en évidence de nouveaux éléments d'explication.

Actuellement, leur vie clandestine et la façon dont sont réalisées les "besognes " disqualifient les tueurs à gages qui jouissaient auparavant d'une certaine reconnaissance sociale. Cette disqualification est directement proportionnelle à la prolifération des actes des meurtriers à louer et à la variété de motif des demandes qui leur sont adressées.

1. L'expression fait référence à un mode particulier de "liquidation de témoin gênant " appliqué aux "traîtres ", cf. infra.

2. La fiction est surtout alimentée par les romans et les feuilletons télévisés mais aussi par les articles de journaux et les récits policiers qui décrivent les fuites spectaculaires des tueurs à gages et le nombre surprenant d'homicides qui leur sont attribués. 
Les tueurs à gages tentent d'arborer des qualités personnelles de courage, d'intrépidité, et d'habileté dans l'usage des armes à feu. Cette dimension émerge des récits des tueurs à gages eux-mêmes. Leur discours oppose la témérité et le courage, et bien qu'ils se réclament de la seconde de ces valeurs, cette référence connote la culture sertaneja ${ }^{1}$, c'est-à-dire la culture rurale. "Je ne suis pas téméraire. Le téméraire est celui qui se bat au couteau. Moi, j’ai le courage de tuer. " À ce point donc, apparaissent des attributs personnels comme le courage, la témérité et la ruse. Dans l'organigramme des meurtriers sur commande existent des vides qui doivent être comblés par des personnes qui possèdent les caractéristiques du courage et de la témérité.

Ainsi, on peut dire que la trajectoire des tueurs à gages comporte un soutien social qui les valorise. Les valeurs de la vengeance, de la témérité et du courage fournissent aux actes des tueurs à gages la base d'une légitimité sociale. Ces actes prennent sens dans une société où il existe une certaine acceptation, une démonstration de sympathie pour les pratiques violentes dans la manière d'affronter les conflits sociaux.

Les meurtriers que la presse et les organes de sécurité désignent comme des "meurtriers à louer " tentent de justifier leurs actes en se plaçant dans la condition de vengeurs ou de justiciers, et non dans celle de tueurs à gages, dépourvue de valorisation sociale. Dans cet article, la place centrale est occupée par la lutte symbolique entreprise, dans leurs récits, par les tueurs à gages pour la qualification de leurs actes.

Les recherches que j'ai réalisées sur les meurtriers à louer et les crimes sur commande (Barreira, 1998) m'ont conduit à percevoir que ces pratiques n'ont plus grande incidence dans le milieu rural mais qu'elles se manifestent principalement dans les grandes villes.

La forme diffuse selon laquelle elles se produisent renvoie à un vaste champ d'affrontement ou de gestion de petites querelles, comme des querelles de voisinage ou des mésententes familiales, qui incluent également la gestion des disputes politiques et économiques. Les motifs de passation des contrats des tueurs à louer sont définis en amont dans leur quasi-totalité par des querelles de ce type.

De telles pratiques m'ont amené à réfléchir sur les nouvelles configurations de l'action des meurtriers à louer au sein des relations conflictuelles.

1. Qui est originaire du "sertão ", terme désignant l'intérieur du pays et la campagne peu peuplée, par opposition à la ville. 
Ces préoccupations sont jalonnées par les actes des tueurs à gages, justiciers et vengeurs, ainsi que par les affrontements violents des conflits sociaux. Ceux-ci questionnent la « cordialité brésilienne » en tant que partie d'un imaginaire issu du sens commun et principalement alimenté par certains secteurs de la classe dominante.

LE MEURTRE SUR COMMANDE :

UNE NOUVELle SilhouetTe DANS DE VIEUX VÊTEMENTS.

Au cours des dernières décennies du $\mathrm{Xx}^{\mathrm{e}}$ siècle, les crimes sur ordonnance se diversifient et adoptent de nouveaux contours. Ainsi qu'il a été souligné précédemment, ils cessent d'être un phénomène éminemment rural et se mettent à occuper des espaces dans le paysage urbain. Les grandes villes qui permettent une fuite plus facile et un anonymat plus grand deviennent les scènes favorites de ces crimes. En tant qu'éléments des meurtres sur commande, les « célèbres chevaux " cèdent la place aux "puissantes motos " dont les casques font d'excellents camouflages. Dans ce cas, une parfaite symbiose s'opère entre les traits sous lesquels le sens commun brésilien se représente le rural-attardé et les traits de l'urbain-moderne. Dans la configuration actuelle, l'utilisation du tueur à gages ne se limite plus à l'élimination des adversaires du donneur d'ordre dans la dispute pour la représentation politique ou dans la résistance autour de questions foncières. Si le tueur à gages occupe aujourd'hui une place centrale dans les affrontements de conflits sociaux, c'est en relation à la sociabilité citadine.

À la fin du XIX et au début du XX ${ }^{\mathrm{e}}$ siècle, les exécutants des « besognes » étaient connus au Brésil et principalement dans la région du Nordeste comme capangas ou jagunços ${ }^{1}$. Ils ont gagné leur notoriété en exécutant leurs "travaux" sous le commandement des grands propriétaires terriens dans le but de régler les litiges avec des familles ennemies et les "litiges " avec des familiers et des ouvriers agricoles.

À cette époque, le commanditaire - l'auteur intellectuel - à qui les tueurs à gages refusent le qualificatif "courageux » exerçait la fonction de financier des délits et de protecteur de l'auteur matériel du crime. De façon emblématique, il incorporait les valeurs d'une culture dominante, définie par des pratiques de protection qui pouvaient être désignées comme clientélistes.

La protection était en relation directe avec la "loyauté », terme désignant la complicité existante à l'intérieur du «monde du

1. Hommes de mains à la solde des grands propriétaires terriens. 
meurtre sur commande ». Il existait une loyauté "absolutisée " ou idéalisée, liée de façon ombilicale à la protection traditionnelle qu'offraient les potentats d'autrefois. Cette loyauté était maintenue entre les tueurs à gages et les protecteurs ou commanditaires de façon directement proportionnelle à la protection que les seconds pouvaient offrir; de telle sorte que l'expression «délivre-toi de la mort et je te délivrerai de la police " en devenait le paradigme. Cette maxime était attribuée aux puissants commanditaires.

Actuellement, la peur de mourir est la composante majeure de la loyauté et de l'absence de dénonciation du nom du commanditaire. Dans le cas contraire, la mort est l'éventualité la plus plausible. La dénonciation entraînerait aussi la perte de tout appui juridique mandaté et financé par l'auteur intellectuel du crime. Aujourd'hui, le silence sur le nom du commanditaire et de l'intermédiaire place la loyauté à un autre degré : celle-ci fait partie des règles de survie du système du meurtre sur commande. La "trahison ", ainsi qu'est généralement désigné l'acte de dénonciation du commanditaire est toujours punie par la mort du traitre. On retrouve ici la figure biblique du Judas, qui dans les représentations liées au folklore populaire doit être pendu et brûlé sur le bûcher en place publique. Cette pratique létale est utilisée pour punir un «traitre " mais aussi pour éliminer un éventuel dénonciateur pouvant mettre en échec le réseau du meurtre sur commande. Cet acte, désigné par les organes de sécurité comme "brûlage d'archives » évoque les pratiques de la mafia italienne.

Les dimensions de fidélité, de confiance et de loyauté sont constitutives de relations fortement marquées par des traits personnels. Elles perdent du terrain au fur et à mesure que les tueurs à gages et les relations du meurtre sur commande se professionnalisent et occupent les espaces urbains.

$\mathrm{La}$ "loyauté » des auteurs matériels du crime vis-à-vis des commanditaires se construit de façon différente selon les réseaux sociaux, la nature des actes délictueux et leurs motifs.

Actuellement, la professionnalisation du tueur à gages est l'une de ses caractéristiques principales. En plus de la perte de ses liens avec un patron, celui-ci s'éloigne des activités économiques, notamment agricoles. Ainsi les activités du tueur à gages sont-elles gérées à l'intérieur du système du meurtre sur commande, à l'intérieur d'un réseau qui dépasse les limites non seulement de la propriété rurale, mais encore du seul État, voire de la région ${ }^{1}$.

1. La Fédération des États-Unis du Brésil est regroupée en cinq grandes régions administratives. 
Les grandes propriétés rurales ont cessé d'être le principal lieu d'habitation et de refuge des tueurs à gages, qui pour la plupart commencent à habiter, et à se protéger, à la périphérie des grandes villes ou dans les dénommées " cités-dortoirs".

La perte de ce lien avec un propriétaire rural particulier, à la fois patron et protecteur ne permet pas de considérer cette catégorie comme délimitée dans l'espace ; ce que ne permet pas non plus sa dispersion dans l'espace et la prolifération de ses activités. Pour les institutions policières, tout cela entraîne la nécessité d'autres formes de classification :

"Auparavant, quand un meurtre sur commande se produisait, la police avait déjà au moins une indication concernant l'auteur matériel et l'exécuteur. Aujourd'hui, les meurtres sur commande ont pris une nouvelle allure. Il y a une vulgarisation. Ceux qui pratiquent actuellement le meurtre sur commande sont des criminels qui agissaient dans d'autres types de délits, comme les attaques de banques et le gangstérisme. Aujourd'hui, le meurtre sur commande s'est déguisé. Autrefois, le tueur à gages se mettait une cagoule sur le visage et il sortait à cheval. Aujourd'hui, il est citadin et il utilise une moto et un casque. Cela rend le travail de la police judiciaire beaucoup plus difficile" (Témoignage d'un commissaire, publié par le journal Diário do Nordeste, le 11 juin 2007).

La passation du contrat avec un tueur à gages peut être réalisée directement par le commanditaire du crime ou bien médiatisée par une autre personne. Actuellement la passation du contrat pour une "besogne" est faite généralement par un intermédiaire, connu comme "courtier de la mort». La présence de cet intermédiaire représente l'institutionnalisation d'un syndicat du crime pourvu d'une hiérarchie et d'une différenciation des rôles. L'existence du « courtier de la mort " permet l'anonymat de l'auteur intellectuel du crime, ainsi qu'une "professionnalisation" croissante du tueur à gages.

La dispersion spatiale des tueurs à gages permet également un accroissement constant de la fonctionnalité des intermédiaires, qui deviennent les rouages essentiels de l'établissement d'un réseau complexe de rôles et de fonctions. Un contrat peut être passé avec un tueur à gages de l'État du Ceará pour un crime dans l'État de São Paulo et vice versa, ce qui donne lieu à un phénomène de migration des crimes sur ordonnance. Les "courtiers de la mort» détiennent désormais les cartes maîtresses du jeu, parmi lesquelles figurent les qualités ou les attributs personnels des tueurs à gages : le courage et la dextérité. Semblables qualités définissent la place de leur possesseur sur le "marché du travail » en les rendant aptes, ou non, à exécuter des services déterminés, plus ou moins dangereux ou complexes. 
L'élargissement et la prolifération des actes des meurtriers à louer configurent à leur tour deux nouvelles pratiques : l'échange de faveur entre meurtriers et l'apparition de la sous-traitance :

«L'échange de faveur se produit de la façon suivante: "Écoute, je suis très recherché dans cette région. Alors, tu tues pour moi ici, et je tue pour toi là-bas." Dans ce cas, la récompense est l'échange de faveur, longtemps en vigueur au Ceará. Il y a aussi la sous-traitance du meurtre sur commande... C'est ainsi : la personne va dans un lieu particulier passer un contrat avec un tueur à gages, mais il [le tueur] dit qu'il a déjà une très grande demande de "besognes". Alors, il sous-traite la besogne pour qu'on fasse le travail pour lui. Il sert [dans ce cas] d'intermédiaire " (journal O Povo, 2 décembre 2004).

Les pratiques de meurtre sur commande adoptent donc d'autres contours conformés par la professionnalisation des meurtriers à louer, l'urbanisation et l'accroissement du nombre de ces actes.

\section{NOUVEAUX RÔLES: RUPTURES ET CONTINUITÉS}

L'élargissement de l'univers des actions pratiquées par les criminels à louer ainsi que leur forte insertion dans les conflits interpersonnels redéfinit les espaces sociaux et économiques des principaux personnages du système du meurtre sur commande. Jusqu'au milieu du $\mathrm{XX}^{\mathrm{e}}$ siècle, les crimes rémunérés étaient pour la plupart circonscrits aux bagarres pour la représentation politique et aux disputes pour la terre, traversées en grande partie par des «querelles de familles ". La terre et le vote apparaissaient comme les principaux ingrédients et les éléments déterminants de l'usage du meurtre sur commande (Barreira, 1998).

Au cours des dernières décennies, des changements de caractéristiques affectent la figure du commanditaire. Auparavant, le commanditaire était principalement le " colonel ", le grand propriétaire terrien qui utilisait les services de tueurs à gages pour résoudre ses "problèmes " avec des familiers et des petits paysans vivant sur ses terres, ou avec des propriétaires de terres en litiges. Les différents conflits politiques, qui impliquaient en général une lutte pour la domination ou le contrôle politique d'une municipalité ou bien la conservation d'une clientèle politique étaient aussi facilement résolus par les «travaux » des tueurs à gages.

Outre des propriétaires terriens et des politiciens, il existe actuellement beaucoup d'autres commanditaires, à savoir les maris jaloux, les entrepreneurs et les commerçants. L'usage des "services » du meurtre sur commande dans la résolution de conflits à l'intérieur 
du champ économique est vraisemblable. Une autre donnée nouvelle est constituée par l'apparition de femmes commanditaires, dont la cible principale est leur mari :

«Le commissariat de la police métropolitaine de Caucaia a révélé hier un autre meurtre sur commande, survenu dans cette municipalité le 6 juillet de l'an dernier: l'assassinat du mécanicien de l'entreprise d'autocar Santa Maria, Antônio Adalberto Nogueira, 53 ans, atteint par une balle. C'est sa propre femme, Fernanda Maria Ponciano, 41 ans, avec qui il était marié depuis vingt-deux ans qui l'a fait tuer, selon ce qu'elle a avoué à la police en alléguant que Nogueira la maltraitait constamment ainsi que ses enfants.

"D'après ce qu'a élucidé la police, Fernanda Ponciano a payé au tueur à gages José Sérgio la somme de 1500 reais pour l'exécution de la "besogne". Elle dit dans son témoignage qu'elle ne supportait plus d'être battue par Antônio Adalberto " (journal O Povo du 18 mars 2005).

Les disputes amoureuses ou les conflits domestiques apparaissent comme un espace d'action plus récent des tueurs à gages.

Au début de la décennie de 1980, dans les zones rurales du nord du pays et dans l'hinterland du Nordeste les pratiques délictueuses qui existaient depuis le $\mathrm{XIX}^{\mathrm{e}}$ siècle reviennent sur le devant de la scène car les tueurs à gages sont appelés à développer et promouvoir l'expulsion des petits paysans de l'intérieur des propriétés. Ils assassinent également des dirigeants paysans potentiels ou effectifs ainsi que des agents pastoraux ${ }^{1}$.

Actuellement, les "carrières » des tueurs rémunérés se construisent principalement dans des contextes de relations moins personnelles et elles s'étendent vers d'autres États et régions du pays. L'incorporation de "valeurs urbaines " élaborées par une expérience de vie dans les grandes villes se dégage de leurs trajectoires. Le profil récent des tueurs à gages présuppose une accumulation d'expériences. Ils ont auparavant commis de "petits délits ", ou bien ils ont participé à des groupes d'extermination dans les grands centres urbains du pays, ou encore commis des assassinats. Graduellement, ces pratiques délictueuses ont mis ces personnages en retrait de toute vie légale en les plaçant dans un réseau d'actes de meurtre sur commande.

Dans l'univers symbolique des crimes sur ordonnance se conjuguent deux aspects : d'une part la disparition des "grands tueurs" connus pour leurs "prouesses " et pour les dizaines de crimes commis, et, d'autre part, la prolifération actuelle des tueurs à gages. La multiplication des "professionnels » confirme le discours des organes de sécurité qui affirment que : "Aujourd'hui n’importe quelle

1. Entre 1984 et 1986, 13 ouvriers agricoles ont été assassinés par des tueurs à gages sur ordre de grands propriétaires ruraux dans le seul État du Ceará. 
petite frappe est tueur à gages. " Petite frappe est entendu au sens explicite de vulgaire, mesquin et méprisable.

Naturellement, les tueurs à gages peuvent apparaitre et élargir leur rayon d'action dans les fissures d'un monopole désordonné de la violence, dans lequel existent des espaces de gestion des conflits interpersonnels, politiques ou économiques qui outrepassent les pratiques institutionnelles.

Si l'utilisation de tueurs à gages dans les assassinats d'adversaires politiques démontre l'importance du vote comme soubassement du pouvoir, si l'assassinat de paysans montre l'importance revêtue par la propriété terrienne et laisse transparaitre la voracité des stratégies utilisées pour la maintenir, l'élimination d'un adversaire dans un conflit interpersonnel révèle en revanche un quotidien violent, marqué par l'absence de dialogue et de négociations. De telles pratiques laissent transparaitre les fissures du monopole de l'exercice de la violence physique qui, selon Max Weber, définit l'État moderne. Elles laissent transparaître aussi l'existence de zones non pacifiées, qui montrent les fragilités du "processus de civilisation " (Elias, 1993).

Comme il a été affirmé précédemment, dans les conflits interpersonnels l'éventail est bien plus large. Il peut s'agir d'un voisin, d'une compagne, d'un débiteur. Le facteur déclenchant peut être l'inimitié ou la rivalité du commanditaire ou bien encore l'inclusion dans une chaîne de vengeances.

Dans ce contexte, il est important de souligner le fait que ce ne sont pas seulement les commanditaires et les meurtriers à louer qui subissent des mutations mais aussi les victimes, qui ne sont plus seulement celles des conflits politiques et agraires. Les victimes sont modifiées par le contexte contemporain de violence diffuse et, fondamentalement, par les conséquences de l'utilisation élargie des meurtriers à louer.

Les différentes stratégies symboliques et leurs effets apparaissent comme des dimensions fécondes pour l'analyse des usages du meurtre sur commande. Les crimes liés à la terre, impliquant l'élimination de dirigeants paysans ou d'agents pastoraux paraissent s'insérer dans un contexte de plus grande visibilité. Cette visibilité de l'action, ou des événements qui précèdent le crime a pour corollaire l'intention de "répandre la peur " en une claire démonstration d'affirmation de pouvoir.

Dans la sphère des conflits interpersonnels, les crimes présentent un mélange d'anonymat et de transparence. Ils se déroulent dans un climat de dénonciations et de rumeurs dont le ressort réside dans le fait que la victime et le commanditaire peuvent faire partie du même réseau de relations interpersonnelles et de voisinage. L'anonymat est entretenu par la forme sous laquelle est perpétré l'assassi- 
nat, par l'utilisation de la surprise, de l'embuscade et, principalement, par l'usage d'un tueur qui ne fait pas partie de ce réseau de relations sociales. La visibilité est fournie par le fait que l'action criminelle se produit dans une vague de rumeurs qui anticipe ce qui va se produire, ainsi que par le fait que ni précaution ni subtilité ne sont de mise dans le passage à l'acte.

Un autre aspect important est la claire explicitation des motifs réels du crime, qui désigne ou définit explicitement un possible commanditaire. Une relation conflictuelle entre le commanditaire et la victime est ainsi tissée et explicitée dans un climat de tension sociale qui laisse présager une tragédie annoncée.

D'un côté, le commanditaire et le tueur à gages sont les deux faces d'une même pièce de monnaie, bien qu'ils occupent des positions différentes dans l'échelle sociale et dans la perpétration du crime. D'un autre côté, ces différences laissent transparaître de profondes contradictions. Dans de nombreuses situations, la relation entre commanditaires et meurtriers à louer est très délicate et conflictuelle et elle fait apparaitre les différences sociales. Les tueurs à gages possèdent en effet une parfaite appréhension des dimensions du réseau qui constitue une relation entre dominants et dominés, une relation asymétrique et hiérarchique :

«Je suis écœuré par la société elle-même, parce qu'il n'y aurait pas de crime s'il n'y avait pas de commanditaire. Et les commanditaires sont des gens de la haute, de la haute société. Et je vais être franc avec vous, moi, j'ai déjà voyagé en dehors du Brésil. Il y a beaucoup de députés, de sénateurs, pas seulement d'ici [de l'État] du Maranhão comme [de l'État] du Piauí, là de votre capitale... Ça veut dire que ce sont eux qui m'écœurent. Il n'y en a qu'un qui soit venu ici. Après ça, il a disparu et celui-ci, moi je l'ai enrichi. Il n'avait rien, c'était un sergent de police. Aujourd'hui, il a une bonne maison, une propriété foncière, du bétail et une voiture. Et moi je suis ici avec seulement une tenue de rechange. »

Dans un autre passage, l'informateur relate :

"Le commanditaire doit payer la plus grande part (en référence à la punition). Parce que c'est lui qui a moissonné la vie de cet être humain, ce n'est pas le tueur à gages. Le tueur à gage ne fait qu'exécuter, celui qui détermine la vie c'est lui qui a passé le contrat. Ça veut dire qu'il doit payer [son acte] en restant plus longtemps en prison. Après lui c'est moi, et après moi l'intermédiaire, parce qu'il doit y avoir un intermédiaire entre lui et moi » (Entretien réalisé à la prison de Pedrinhas, dans l'État du Maranhão).

Dans ce récit, le tueur à gages se sent jouer un rôle, un rôle tragique et dangereux mais qui a été échafaudé par une autre personne qui n'a pas «le courage de réaliser, qui ne veut pas voir son nom exploser dans les pages des journaux. Alors il va contracter un tueur 
à gages ". Dans cet extrait, la différenciation de rôle et d'attributs personnels distincts est clairement affirmée. Le tueur à gages se place comme exécutant une action dont la volonté est située hors de lui-même, ce qui diminue sa culpabilité.

Vis-à-vis des "victimes ", le tueur à gages établit une relation de parfaite neutralité et distanciation. Dans cette relation, les problèmes qu'il résout n'étant pas les siens mais ceux d'autrui, il n'y a pas non plus de place pour des sentiments de haine ou de peine.

Dans cette relation de neutralité devant la "victime ", le tueur à gages recherche la reconnaissance de ses talents individuels par le biais de qualités particulières : la bravoure, le courage et l'habileté personnelle.

Le tueur à gages est la pièce maîtresse d'un jeu solidement enraciné dans une histoire formée par des disputes personnelles résolues hors de l'espace institutionnel et légal. L'usage du meurtre sur commande dans la résolution de certains conflits, principalement ceux qualifiés de "conflits interpersonnels " apparaît comme la négation de la parole, l'impossibilité du dialogue, construisant la place de la violence et des balles.

Dans les récits des informateurs, les défaillances de la justice devant certains cas apparaissent comme justifiant pour une bonne part les pratiques de meurtres sur commande: "À l'époque (où l'une de ses sœurs a été violentée) la justice n'a rien fait contre les criminels et ça m'a révolté. Si la justice ne résout rien, qui est-ce qui va résoudre? Celui qui a de l'argent ne va pas en prison. Il n'existe que deux justices : celle de Dieu et celle du 38 (numéro du calibre d'une arme à feu) dans la tête. " Dans un autre passage de l'entretien, le même personnage confirme : "Je crois que la plupart de ces choses arrivent parce qu'il n'y a pas de justice. "

Dans les trahisons, les vols et même dans la résolution de conflits entre des parties en litiges, les tueurs à gages occupent, partiellement, la place du «justicier ». Cette place qui apparaît dans les récits pour diminuer le "sentiment de culpabilité » sert aussi à expliquer socialement le rôle du tueur à gages. En effet, elle crée une classification morale, mais aussi sociale, des crimes.

Dans un autre entretien réalisé avec un inculpé de meurtre sur commande, celui-ci affirme qu'il s'est "fait une réputation " parce qu'il a tué une personne en état de légitime défense. Selon lui, cette victime a tué un couple de vieillards et elle passait son temps à créer des problèmes. "Il devait mourir, mais je l'ai tué en légitime défense ". Il affirme ensuite : "Je crois que les gens m'aiment bien parce que j'ai tué une personne qui avait tué un couple de vieillards. " Ici apparaît à nouveau la figure récurrente du vengeur. Et dans ce cas, un "couple de vieillards " considérés dans notre société 
comme sans défense est "sacré ". Il est important de relever que les crimes contre les personnes âgées appellent dans l'échelle morale une peine élevée, proportionnelle à l'acte. Dans cet acte, la vengeance est alimentée par la nécessité de pratiquer une asepsie du champ social.

Parmi les justifications que le tueur à gages donne de ses actions figure la valorisation de ses attributs personnels, dans laquelle luimême occupe la place de celui qui a le courage de réaliser une action dangereuse. Le tueur à gages et le commanditaire apparaissent donc comme parfaitement complémentaires dans le système du meurtre sur commande : l'un détient le pouvoir et l'argent, l'autre le courage et les capacités.

Une typologie des tueurs à gages peut être dressée, basée sur la définition de trois situations: traditionnelle, occasionnelle et professionnelle moderne. Cependant, il n'y a pas de "type pur» et la plupart du temps se produit une parfaite symbiose entre les trois. Le modèle traditionnel est très marqué par le passé. Établi la plupart du temps à l'intérieur d'une propriété rurale, le tueur est protégé par un propriétaire terrien particulier. Toutefois, cela ne l'empêche pas de réaliser des besognes à l'intérieur d'un réseau plus ample qui implique des intermédiaires et un rayon d'action plus large. Ce tueur à gage traditionnel vit dans l'ambivalence entre l'ancien et le moderne, entre une protection personnalisée et une protection diffuse qui découle de la possibilité de professionnalisation de ses services. Ce type subit un processus de disparition rapide, qui résulte des divers aspects précédemment analysés.

Le tueur à gages qui réalise des services occasionnels possède une autre insertion professionnelle. Il mène une vie de légalité qui procède de l'anonymat complet de ses actions. Il est peu connu à l'intérieur même des réseaux du meurtre sur commande, mais il est toujours disponible pour un service de plus. Il réside en général à la périphérie des grandes villes tout en étant originaire de milieu rural où, sauf exception, il a pratiqué son premier assassinat. Les «besognes » réalisées par ce type de tueurs à gages ne passent pas forcément par des intermédiaires, le contact étant directement établi par le commanditaire. Le tueur à gages entre dans le réseau du meurtre sur commande par d'anciens tueurs qui transferent, "sous-traitant » les besognes à des nouveaux.

Le troisième type est le tueur à gages dont le «travail " est totalement professionnalisé. Il appartient au réseau du meurtre sur commande et sa principale activité est le crime commandité. Toute autre insertion professionnelle n'intervient que comme camouflage afin de permettre la réalisation de son activité principale tout en détournant d'éventuelles suspicions des organes de sécurité. Un tueur à gages qui se présentait comme tel affirmait qu'après une 
"action dangereuse », il travaillait comme chauffeur de camion jusqu'à ce qu'il soit oublié des organes de sécurité. La protection de ce type de tueurs est parfois assurée par des accords clandestins entre les commanditaires et les organes de sécurité qui comptent également une certaine complicité de la justice. Ce tueur à gages est inséré à l'intérieur d'un réseau où pouvoir économique, pouvoir policier et pouvoir judiciaire sont présents. Le tueur, rouage de ce complexe "jeu de la mort " ne possède pas de domicile fixe et fait du nomadisme sa protection. Il est constamment en déplacement pour accomplir des «besognes» dans différentes parties du pays.

Les trois types de tueurs se présentent comme disponibles pour réaliser n'importe quel "crime sur commande". Toutefois, les intermédiaires font le tri ou la sélection en mettant en relation les attributs personnels des tueurs avec la nature du crime ou la position sociopolitique de la victime.

\section{VENGEANCES ET CONFLITS INTERPERSONNELS}

Dans les actes délictueux pratiqués par les tueurs à gages, la différence entre crime de vengeance et crime rémunéré est très ténue. La trajectoire de ces hommes se constitue dans cette ambivalence entre tueur à gages et vengeur, qui résout ses propres problèmes de querelles personnelles, et ceux des autres, sur commande. En ce sens, le "monde du meurtre sur commande" ne nous fournit pas de "type pur » de tueur à gages, de même qu'une affirmation du type "je ne suis pas un tueur mais un vengeur" n'est pas tout à fait vraie.

Il n'existe pas de conduite univoque : on tue par fidélité ou gratitude vis-à-vis d'un commanditaire éventuel et l'on tue aussi pour de l'argent, tout comme l'on tue un adversaire personnel. Actuellement, les relations d'échanges médiatisées par des éléments comme la fidélité et la gratitude perdent du terrain au profit des échanges mercenaires, qui configurent une autre relation entre problèmes interpersonnels et argent, ou, en d'autres termes, entre les querelles personnelles et celles d'autrui.

Les tueurs à gages apparaissent donc comme des porteurs de solutions aux problèmes privés et aux disputes entre rivaux. Ils seraient la "main armée " des personnes dont le droit serait bafoué ou supposé tel. Les tueurs à gages apparaissent comme des vengeurs et des redresseurs de torts pour autrui, par l'intermédiaire de solutions violentes. De telles solutions sont, en partie, socialement légitimées dès lors qu'existe une demande sociale et que l'opinion publique est mobilisée pour disqualifier la victime : policier cor- 
rompu, femme infidèle, commerçant malhonnête, entrepreneur sans scrupule.

Dans son récit, un informateur fait valoir que «les personnes de ma famille sont vindicatives et sont nées comme ça ». Cependant, il souligne la différence entre bandits et hommes vindicatifs. "Dans ma famille, il existe des hommes de biens, qui sont médecins, ingénieurs, généraux et députés. Ce sont des bagarreurs, mais pas des bandits. »

Dans une autre partie de l'entretien, il affirme que «le tueur à gages tue pour de l'argent et le vengeur a de l'amour pour son sang, car quand il voit son sang versé, il veut aussi voir celui de l'autre versé ». Ce même informateur dit aussi que «le meurtre sur commande ne s'arrête jamais parce qu'il y circule de l'argent, et que quand un type est affamé et qu'il ne sait plus quoi faire, il cherche tout de suite une solution à son problème».

Au sein des luttes pour la classification symbolique des pratiques délictueuses et parmi les accusations portées par les organismes de sécurité publique, l'interviewé se présente comme un bouc émissaire des crimes perpétrés dans le Nordeste au cours des décennies de 1970 et 1980 ; crimes que la police ne voulut pas ou n'arriva pas à élucider. Pour la police, ce personnage est considéré comme le plus grand tueur à gages du Nordeste. Près de 80 homicides lui sont attribués. Dans sa propre classification et dans les désignations qu'il fait de ses propres crimes, il dit que tous :

«furent commis dans le champ de l'honneur. Si on me traite de voleur, je tue, car je ne suis pas un voleur. Le procureur dit que ce n'est pas une raison. Je lui ai rétorqué que c'est une vraie raison, et je lui ai dit que si lui était un voleur moi je ne l'étais pas et que je ne regrettais pas ce que j'avais fait pour mon patron. Moi, je lui ai dit que si un homme le tuait, je le vengerais par la mort ».

Dans les récits des tueurs à gages prédomine un discours de négation de leurs liens au "monde du crime " et plus spécifiquement au système du meurtre sur commande. À l'intérieur de la culture sertaneja ${ }^{1}$, il existe un adage qui dit qu'il est préférable d'être assassin plutôt que voleur, ou, en d'autres termes, que l'acte de voler entache l'honneur d'un homme, tandis que tel n'est pas le cas de l'assassinat.

Ainsi, ce qui transparait dans la pratique de l'homicide est le fait que pour l'homme rural, l'honneur ne réside pas dans l'ensemble des valeurs et des interdits qu'il doit être capable de défendre mais 
«dans le comportement violent qu'il ne doit pas hésiter à adopter pour les défendre "(Fatela, 1989, 57).

Dans le discours des personnes interviewées précédemment mentionné, la société est représentée au moyen de relations conflictuelles générées par la jalousie, par la concurrence mais aussi par les différences sociales.

Le climat d'insécurité et d'inquiétude produit la nécessité que des personnes occupent la place de gardien ou de vengeur. La "jalousie " apparaît comme une catégorie fondatrice des relations sociales et instigatrice des conflits interpersonnels. Sans espace pour les fragilités, la violence est naturalisée et il n'existe alors de place que pour le courage et la ruse. Le respect apparait comme un synonyme de la peur et comme une démonstration de fragilité.

Les crimes assumés par de potentiels meurtriers à louer représentent une proportion bien plus faible que celle indiquée par la police. Ils sont principalement perpétrés à l'intérieur du «champ de l'honneur " et marqués par un fort aspect de "vengeance ". En ce sens, la police ou la justice apparait comme coupable des assassinats commis, par omission ou par incompétence :

"Je n'ai fait que me venger. S'il y avait la peine de mort, celle qui s'occuperait de la vengeance, ce serait la loi. Maintenant avec cette police-là ne vont mourir que des pauvres et des innocents. S'il existait la peine de mort, ceux qui ont tué mon père et ma sœur seraient morts et moi, je ne serais pas ici et je serais docteur. Je pense que la majeure partie des choses arrive parce qu'il n'y a pas de justice »" (entretien réalisé au Centre pénitentiaire Paulo Sarasate, État du Ceará).

D’un côté, les récits des personnes fortement présumées d'avoir participé à certains meurtres sur commandes et même à des réseaux font valoir une négation des valeurs présentes dans le "monde du crime ". D'un autre côté, ils mettent en avant les valeurs de bonté, de compassion, de désintéressement et de sensibilité. Avec une grande récurrence apparaissent les figures du bon fils, du bon père, du bon mari, ainsi que celle de l'ami loyal et solidaire, tous traits culturels qui récusent une possible assimilation au tueur à gages.

Un autre informateur, détenu pour meurtre sur commande a nié toute implication dans des crimes sur ordonnance. En donnant sa propre version de ses attributs et en mettant en valeur leurs aspects religieux, il affirme :

"Je ne suis pas différent des autres personnes. Ave Maria! Je suis un être humain! Je suis un bon copain qui se fait facilement des amis. Je voudrais que vous alliez là où $j$ 'habitais pour savoir quel est mon caractère, et

1. Détenu accusé de pratiquer des meurtres sur commande, condamné à trente années de réclusion. 
que vous demandiez si un jour j'ai payé des sodas, des mangues, du jus de cajou aux enfants. Parfois je prie et je demande à l'ange gardien de ces enfants dont je rassasiais la faim qu'il intercède auprès de Dieu pour moi. Je le faisais en pensant à eux. Les gens se trompent beaucoup à notre sujet. Mon cœur est très bon, vous le croyez? Je pleure pour un enfant pauvre. Ça ne me fait rien de demander l'aumône, mais quand je vois un enfant qui a faim, je partage ma nourriture avec lui. Je crois qu'un jour, j'atteindrai la liberté " (Entretien réalisé au Centre pénitentiaire Paulo Sarasate, État du Ceará).

Comme il a été dit précédemment, dans leur majorité, les homicides envisagent leurs crimes comme des "vengeances". Ils nient ainsi pratiquer des "meurtres sur commande " et effacent le caractère froid et impersonnel du " crime sur ordonnance " pour le seul argent. En ce sens, il s'agit d'une tentative de mêler à l'assassinat des motifs affectifs, moraux et émotionnels. Le suspect cesse d'être "froid, calculateur et inhumain ", et, à l'inverse, construit un personnage plein d'émotions socialement reconnues comme justes.

Dans cette logique, les meurtres sur commande peuvent explicitement avoir la vengeance comme ingrédient ou comme motif. La vengeance apparaît alors comme effaçant son caractère mercenaire au meurtrier et peut dans le même temps apparaître aussi comme le ressort déclencheur du meurtre sur commande.

Un cas, relaté par le journal O Povo du 17 mars 2007 dans un article intitulé "Victimes de la haine " est paradigmatique de la symbiose entre vengeance et meurtre sur commande. Les faits se déroulent sur plusieurs années et peuvent être résumés ainsi :

- En février 2004, Jairo Barreto Sobrinho est assassiné par balles à Jaguaretama. D’après la police, le crime aurait été planifié par Luciano Saraiva Diógenes, qui se trouvait détenu à l'époque du crime. Selon un familier de la victime, cette dernière aurait fait une plaisanterie sur un membre de la famille Diógenes pendant une beuverie.

- En juillet 2005, Luciano Diógenes est assassiné. Les soupçons se portent sur un homme connu sous le nom de Chiquinho, qui entretiendrait une relation amoureuse avec la femme de la victime.

- En septembre 2005, Jeová Barreto, 68 ans, père de la première victime est tué de six coups de pistolet dans le centre du bourg de Jaguaretama par un homme qui s'enfuit à moto. Quelques mois plus tard, Chiquinho est trouvé mort par balles.

- En février 2007, Ana Mônica Barreto, sœur de la première victime est tuée de huit coups de pistolet en rentrant de son travail 
par deux hommes à moto. Deux personnes liées à la famille Diógenes sont désignées par la police comme étant les auteurs de l'exécution.

Les disputes familiales qui ont marqué profondément la région Nordeste du $\mathrm{Xx}^{\mathrm{e}}$ siècle ont déjà été analysées par Luis Costa Pinto (1980), Billy Chandler (1980) et beaucoup d'autres. Les détails du résumé ci-dessus montrent la récurrence des rivalités entre familles au Brésil. Ce cas, qui montre la reconfiguration de ces disputes familiales en plein $\mathrm{XxI}^{\mathrm{e}}$ siècle, implique les Barreto et les Diógenes, deux familles de la commune de Jaguaretama, État du Ceará, située à $245 \mathrm{~km}$ de Fortaleza, la capitale. Ce sont des pratiques anciennes comportant de nouvelles ressources, parmi lesquelles apparait l'image de la moto, modalité toujours présente dans les meurtres commandités plus récents. L'article du journal met en avant le fait qu'il s'agit d'une sanglante rivalité familiale, entretenue par un réseau de soutien qui dépasse les limites d'une simple commune et articule un réseau de protection des crimes de sang. Une donnée importante de ce communiqué consiste dans ce que pour la police, les tueurs à gages puissent être membres d'une " famille importante, économiquement et politiquement ". Cette donnée signale deux aspects : elle démontre tout d'abord l'existence d'une hiérarchie et d'une division de rôles socialement différents à l'intérieur d'une famille, tout comme la difficulté analytique de séparer la pratique du meurtre sur commande de celle de la vengeance. Ensuite, elle démontre à nouveau de façon claire que les tueurs sur commande peuvent résoudre tant leurs propres problèmes que ceux d'autrui.

\section{CONCLUSION}

L'utilisation de tueurs rémunérés dans la gestion ou l'affrontement des conflits sociaux montre que les pratiques de meurtres sur commande se réarticulent dans un cadre plus récent. Sous un nouvel habillage et des traits contemporains, la base d'une telle configuration est définie par des traits anciens qui reproduisent d'anciennes pratiques. Dans ce cadre les fonctions des tueurs à gages s'étendent et gagnent du terrain. Elles cessent d'être limitées à la problématique rurale, aux luttes politiques médiatisées par les conflits familiaux ou à la lutte pour le maintien du monopole politique et économique. L'élargissement de ses fonctions ôte son caractère éminemment rural à cette pratique. Par le passé, le tueur à gages entretenait une relation forte avec un grand propriétaire terrien visà-vis duquel il exerçait d'ailleurs les fonctions de travailleur agricole ou de familier dans la propriété foncière. Le commanditaire et 
auteur intellectuel du crime cesse d'être ce propriétaire rural, le célèbre «colonel» ou le potentat politique. Ces modifications dans le profil social et économique du meurtrier sur commande tout comme dans celui du commanditaire rendent impossible pareille définition préalable de ces personnages. Pour la police, n'importe quel petit voyou peut actuellement exercer la fonction de tueur à gages. Au sein d'un éventail élargi des motifs de meurtres, le commanditaire n'appartient plus nécessairement à la classe dominante. Dans ce cadre de redéfinition, la victime subit aussi des changements et peut être un "simple adversaire".

La récente configuration des pratiques de meurtre sur commande conduit à l'élargissement des classifications symboliques et des représentations sociales relatives à la compréhension de la signification des actes sociaux des tueurs rémunérés. Concernant un possible conflit de signification, une donnée récurrente est la tentative des tueurs à gages d'ôter le caractère "froid et cruel » de leurs actes en les plaçant sur le plan de pratiques socialement et moralement acceptées et expliquées. Sur ce plan, ils s'expriment comme des vengeurs ou des justiciers qui exercent la fonction socialement acceptée «d'éliminateurs de personnes représentées comme indésirables».

À travers le montage des types de personnes qui doivent ou qui peuvent mourir s'élabore la figure d'un justicier, qui a pour fonction de pratiquer une asepsie sociale. Dans un tel montage apparaît une classification morale, selon laquelle doivent être éliminés ou punis les mauvais spécimens, épouses infidèles, travailleurs malhonnêtes qui constituent clairement une typologie possible des victimes : les hommes riches, avares, égoïstes et puissant doivent mourir, "et non les pauvres"; la trahison amoureuse ou économique doit être punie. La punition en question, la mort, en vient à être socialement justifiée et expliquée.

Cet «éventail » s'élargit au fur et à mesure que les éliminations impliquent des conflits interpersonnels. Surgissent alors les «voisins indésirables " et les personnes porteuses de dettes économiques. Mettre en avant qu'un possible repentir de la part de l'auteur matériel du crime quant au motif de l'élimination de l'adversaire soit accepté ou verbalisé serait une idée fallacieuse. Dans cette position, le tueur rémunéré conserve la neutralité de la victime qui renforce au contraire son rôle de réel justicier.

En tant qu'ingrédient d'une bonne partie des crimes et revendiquée comme telle par certains tueurs à gages, la vengeance ne rend pas compte de la diversité des motifs des pratiques létales. Il est important de souligner cet aspect dans la mesure où la quasi-totalité de leurs crimes sont ainsi présentés par la majorité des tueurs rémunérés. 
Au fur et à mesure que le tueur à gages se professionnalise, il se déracine de son groupe. En ce sens, il perd l'une des caractéristiques de la vengeance, à savoir la proclamation de la prééminence du groupe sur l'individu.

Le prestige atteint par le tueur à gages est difficilement détourné en bénéfice social. Un aspect transparait cependant qui crée une ambivalence entre vengeance et meurtre sur commande : le sentiment d'une justice commune. Dans le champ de la vengeance, offenseur et offensé se revendiquent d'un sentiment commun de justice. Dans le champ du meurtre sur commande ce sentiment pourrait-il être revendiqué par le commanditaire, le tueur à gages et la victime? L'argument le plus manifeste est que pour la vengeance, ce sentiment est "socialement donné " tandis que pour le meurtre sur commande il doit être "socialement construit " par la revendication de la force physique comme devenant légitime.

Dans le champ des représentations, les personnes possédant une trajectoire de "hors-la-loi » occupent la place ambivalente de bandit et de héros. En ce sens, le bandit peut être le criminel mais aussi le protecteur, le justicier, le héros.

Dans les valeurs existe une ambiguité qui concerne non seulement la manière dont les bandits sont placés dans la fonction de héros mais encore la fonction de hors-la-loi telle qu'elle est véhiculée par les institutions judiciaires.

Ici entre en jeu l'autoreprésentation du tueur à gages médiatisée par des valeurs de courage et d'intrépidité, qui nie toute inconscience et agressivité en affirmant : "Je suis courageux mais je ne suis pas téméraire." La témérité apparaît comme l'attribut personnel de celui qui tue en duel, de celui qui possède la capacité d'affronter un ennemi. En ce sens les crimes classifiés comme des meurtres sur commande sont doublement niés par leurs auteurs. Ils ne résultent pas de la résolution de conflits interpersonnels, ni de la vengeance, de même qu'ils sont réalisés "lâchement et traîtreusement ».

Sur une échelle idéale et sur le plan de la fiction, le tueur à gages possède suffisamment d'audace pour résoudre les conflits d'autrui, de même qu'il est assez fort pour résoudre une "bagarre " ou une mésentente.

Il reste alors aux tueurs à gages deux options : se positionner comme vengeur ou comme courageux, le courage étant une qualité déniée aux commanditaires. Toutefois, comme il a été montré, les tueurs à gage entrent dans un échange de "dons » : courage contre argent. Le courage est probablement l'attribut personnel le plus estimé par les tueurs à gages, en tant qu'il est un aspect de distinction déterminant dans l'initiation aux meurtres sur commande. Le 
courage, la fermeté devant le danger est un capital personnel. Dans le récit des informateurs apparait clairement une différence entre le courage, synonyme d'audace et de bravoure et la témérité, qui correspond à la force et à la vigueur. Sur une autre échelle idéale, le tueur à gages possède suffisamment d'audace pour résoudre les conflits d'autrui, mais il n'est pas suffisamment fort pour résoudre une bagarre qui exige un engagement physique personnel.

L'action des tueurs rémunérés dans la résolution des conflits, interpersonnels ou impliquant des tiers met en évidence un quotidien violent et constitue, fondamentalement, une violence diffuse.

\section{BIBLIOGRAPHIE}

Barreira C., Trilhas e Atalhos do Poder: conflitos sociais no sertão, Rio de Janeiro, Rio Fundo, 1992.

Barreira C., Crimes por encomenda : a pistolagem no cenário brasileiro, Coleção Antropologia da Política, Rio de Janeiro, Éd. Relume Dumará, 1998.

Black-Michaud J., Cohesive Force : Feud in the Mediterranean and the Middle East, New York, St. Martins's Press, 1975.

Bourdieu P., O Poder Simbólico, Lisboa, Difel, 1989.

Busquet J., Le droit de la Vendetta et les "Paci » Corses, Joué-lès-Tours, Éd. La Simarre, 1994.

Carone E., A República Velha (instituições e classes sociais), São Paulo, Difusão Européia do Livro, 1970.

Chandler B. J., Os Feitosas e o Sertão dos Inhamuns, Fortaleza, Éd. UFC, 1980.

Clastres P., Recherches d'anthropologie politique, Paris, Le Seuil, 1980.

Damatta R., Carnavais, Malandros e Heróis : para uma sociologia do malandro brasileiro, Rio de Janeiro, Éd. Guanabara, $5^{\mathrm{e}}$ éd., 1990.

Elias N., La civilisation des mours, Paris, Calmann-Lévy, 1973.

Fatela J., O Sangue e a Rua : elementos para uma antropologia da violência em Portugal (1926-1946), Lisboa, Publicações Dom Quixote, 1989.

Franco Maria Sylvia de Carvalho, Homens Livres da Ordem Escravocrata, São Paulo, Kairós, 3e éd., 1983.

Gilmore D., Agression and Community. Paradoxes of Andalusian Culture, London, Yale University Press, 1987.

Girard R., La violence et le sacré, Paris, Bernard Grasset, 1972.

Mauss M., Sociologie et anthropologie, Paris, PUF, 1950.

Palmeira, M., Nordeste : mudanças políticas no século XX, in Cadernos Brasileiros, Rio de Janeiro, n 37, ano VII, set.-out., 1966.

Pinto, Luiz de A. C., Lutas de famílias no Brasil, São Paulo, Editora Nacional,

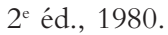

Pitt-Rivers J., Anthropologie de l'honneur: la mésaventure de Sichem, Paris, Le Sycomore, 1983.

Simmel G., Le conflit, Paris, Éd. Circé, 1992. 